\title{
Interest in pelvic packing after hysterectomy for obstetrical haemostasis in maternal rescue: A retrospective study.
}

\author{
F. Ferhi, K. Tarmiz, H. Khouadja, M. A. Bouslama, A. Khlifi*, K. Benjazia \\ Department of Anesthesiology and Critical Care Medicine. Farhat Hached University Teaching Hospital. Sousse Tunisia \\ * Department of Obstetrics and Gynecology. Farhat Hached University Teaching Hospital. Sousse 4002. Tunisia
}

\section{BACKGROUND AND GOAL OF STUDY:}

Severe postpartum haemorrhage is the leading cause of maternal mortality and morbidity worldwide.

Surgical treatment may be indicated and may include a haemostatic hysterectomy in cases of unsuccessful conservative treatments. However, there is a risk of persistent haemorrhage after haemostatic hysterectomy when severe coagulation disorders occur. Pelvic packing can be an alternate life saving technique to control haemorrhage, especially if angiography and embolization is not immediately available.

The aim of this study was to evaluate the possible benefit of the pelvic packing technique after haemostatic hysterectomy associated with coagulation disorders in the immediate post partum period.

\section{MATERIALS AND METHODS:}

We conducted a retrospective survey on hospital records of patients who underwent haemostatic hysterectomy for an obstetric cause.

The study period ranged from January 1998 to December 2016. The files of the selected patients were analyzed for the aetiologies of bleeding, treatment strategies and the indications of packing.

\section{RESULTS:}

During the study period only 32 files were recorded. Caesarean delivery occurred in $84 \%$ (27 cases) of the cases. The aetiologies of bleeding were dominated by uterine atony and retro placental hematoma. Initial medical and surgical management was proved to be insufficient and the transition to a complementary surgical treatment was indicated. Pelvic packing indications were taken collectively. Large compresses were used for Pelvic packing and a drain was left in place in $71 \%$ of cases. The duration of the packing was less than 24 hours in 7 cases, between 24 and 48 hours in 14 cases and between 48 and 72 hours in 13 cases. In one case a surgical revision was necessary after packing in the presence of hemodynamic disorders requiring ligation of the hypogastric artery. Embolization was needed in one case. Postoperative complications were postoperative fever (7 cases), parietal infection (2 cases), postoperative urinary infection (1 case) and deep vein thrombosis ( 1 case).

There were no maternal deaths.

\section{CONCLUSION(S):}

Pelvic packing seems appropriate in case of uncontrollable bleeding after haemostatic hysterectomy associated with persistent coagulation disorders especially if angiography and embolization is not immediately available. In our study, this technique was effective with a success rate of $93 \%$. 